\title{
L'interculturel dans l'éspace francophone
}

\section{Virginia Boza Araya}

Universidad Nacional

Le IXème Sommet de la francophonie a eu lieu a Beyrouth au Liban, les 18, 19 et 20 octobre 2002. Le choix du Liban est hautement symbolique car il confirme la solidarité de la francophonie avec les pays arabes ainsi que l'un des ses principaux rôles qui est celui de favoriser le dialogue des cultures. Ce sommet réaffirme l'intérêt grandissant de beaucoup de pays d'appartenir à cette francophonie même si le français n'est pas leur langue officielle. Pour mieux comprendre cet intérêt il faut tout d'abord cerner le monde francophone et faire un rappel de son évolution.

La francophonie est l'espace de l'interculturel par excellence grâce à la diversité des cultures qui la composent et aux multiples langues qui y cohabitent. Cependant elle commence à peine à se définir comme tel car depuis ses origines c' est le français du nord de la France qui s'impose et qui domine encore la scène francophone. Il faut reconnaître que les moyens mis en oeuvre pour y parvenir ont été particulièrement efficaces. Cette domination a créé tout au long de l'histoire des conflits entre la France et les autres entités qui utilisent le français pour communiquer. Aussi est-elle en mutation car après l'indépendance de la plupart des pays ils se sont trouvés dépossédés de leurs racines et ont dû partir à la quête de leur identité spoliée par la colonisation. Pour essayer de mieux comprendre son mal être et son évolution on fera un bref rappel historique. On s'attachera à analyser les différents conflits qui l'ont tourmentée pour expliquer comment, dans ce contexte, se développe l'interculturel. 


\section{La langue française}

La langue française commence à être reconnue grâce à deux grands événements : le Concile de Tours, en 813, qui autorisait les prêtres à prêter serment en français (langue du peuple) et les Serments de Strasbourg (premier texte rédigé en français) signé en 842 entre Charles le Chauve et Louis le Germanique.

Une série de facteurs vont favoriser la disparition des dialectes et imposer le français comme langue unique. Parmi eux citons: L'ordonnance de Villers-Cotterêt qui imposait, sous François I, le français comme langue juridique en 1539; La Défense et illustration de la langue française de J. du Bellay en 1549 (oeuvre d'une grande importance qui reflète les inquiétudes d' ordre linguistique qui agitaient la Renaissance); la fondation de l'Académie Française (qui avait pour mission la conservation de l'excellence de la langue: Dictionnaire d'usage, Grammaire, Traité de rhétorique) en 1635; L'universalité de Rivarol en 1784. La Révolution française a joué un rôle capital car elle a instauré une politique d'éradication des dialectes et de langues régionales. La création de l'école (obligatoire pour tous les citoyens) au XIX ème siècle permettra au français de s'imposer définitivement comme langue nationale. Finalement en 1992 la Loi Toubon est adoptée afin de limiter l'utilisation de mots étrangers et plus particulièrement des anglicismes. Malheureusement, cette loi n'est pas respectée par la plupart des entités concernées.

Outre ces faits marquants de l'histoire de la langue française; il y a eu la création de différents organismes et/ou associations dont la vocation était celle de promouvoir l'enseignement du français et l'image de la France dans le monde entier. Parmi elles citons l'Alliance Française (1883), la Mission Laïque (1902), l'Association confessionnelle-catholique, l'AUPELF - Association des Universités partiellement ou entièrement de langue française - (1961), l'AUF Agence Universitaire francophone et plus récemment TV5.

L'action de ces institutions est relevante pour la langue française car elles ont créé des foyers francophiles qui sont disséminés un peu 
partout dans le monde. Ces foyers sont devenus par la même occasion des défenseurs de la langue française. En effet, dans beaucoup de pays non francophones le français est enseigné principalement dans des lycées publics et privés, dans les universités et parfois même à l'école primaire, consolidant ainsi l'image du français comme "langue de culture". C'est le cas dans plusieurs pays d'Amérique Latine tels que le Mexique, la Colombie ou le Costa Rica. A cet égard, il faut reconnaître l'excellent travail des Centres Culturels et de Coopération technique des Ambassades de France dans les divers pays en ce qui concerne la formation de professeurs FLE qui perpétuent - par leur travail - la langue, la culture et les valeurs humanistes de la France. Il est vrai que la langue française est en déclin dans certains pays soit parce qu'elle est rejetée en tant que langue du colonisateur soit à cause des assauts de l'anglais qui s'impose de plus en plus dans tous les domaines. Ceci s'explique en particulier par un certain statisme du français qui présente une réticence certaine à accepter des néologismes ou des mots venant des pays francophones quels qu'ils soient.

\section{La Francophonie}

Le mot francophonie a été inventé, en 1880, par le géographe Onésine Reclus dans le but de désigner tous les pays qui pour diverses raisons parlaient le français que ce soit sur le plan interne ou sur le plan international.

Le premier foyer de la Francophonie a été créé un an après la signature des Serments de Strasbourg. En effet, en 843, Charles le Chauve et Louis le Germanique ont octroyé à leur frère Lothaire une région où on parlait le roman (futur français): la Lotharingie. A la mort de celui-ci ses héritiers vont se partager les terres, ce qui explique l'existence d'autres pays francophones en Europe : la Belgique, la Suisse et le Luxembourg.

Aux territoires francophones d'Europe cités auparavant s'ajoutent, au cours des siècles, d'autres régions ou communautés: d'anciens pays colonisés et des communautés utilisant le français comme langue de communication internationale. 
$\mathrm{La}$ francophonie constitue donc un ensemble très complexe qui regroupe, à nos jours, 54 pays. Bien que la langue française soit à la base ce qui les réunit, la place qu'elle occupe dans chaque pays n'est pas identique dû à leur histoire et aux différentes cultures qui y cohabitent. En effet le français parlé au Zaïre ou d'autres pays af ricains n'est pas de même nature que celui de la Belgique ou celui du Canada.

Pour avoir une vision claire de la francophonie il faut donc déterminer le degré et la nature de la présence du français dans chaque pays. Pour ce faire il faut considérer deux critères : d'une part le statut de la langue : langue maternelle, langue seconde (ex. Pays du Maghreb, Madagascar), langue officielle (anciennes colonies d'Afrique noire), langue de culture (ex. la Roumanie où le français est étudié par $35 \%$ des élèves), langue étrangère privilégiée (ex. le Liban où le français s'est implanté par l'intermédiaire des missions, surtout depuis le XIXème siècle. Environ la moitié de la population la pratique) et d'autre part le corpus qui détermine la réalité socio-linguistique. Cette distintion est essentielle car il y a des distorsions entre les différents pays : par exemple le français est la langue officielle du Gabon mais il n'y a que $6 \%$ de la population qui le parle. Il en est de même à Haïti et dans d'autres îles des Antilles et de l'Océan Indien où le créole est parlé par la majorité des habitants. Il en va autrement pour des pays comme l'Ile Maurice dont la langue officielle est l'anglais mais un grand nombre de citoyens parle le français. En tenant compte de ces deux paramètres, ces pays peuventêtre classés en différentes catégories ainsi que le démontre, par exemple, la carte du Haut Conseil de la Francophonie qui privilégie les aspects sociolinguistiques. Par contre la carte diffusée par Hachette classe les pays en fonction du nombre des locuteurs. L'Université de Laval prend plutôt en considération des distinctions purement linguistiques : langue maternelle, langue of ficielle ou administrative (inclut autant les pays africains que les DOMTOM), ou bien comme langue d'enseignement. ${ }^{1}$ Ils peuventégalement

$\overline{1 . \quad \text { Ex. carte }}$ diffusé sur le site $<$ http://www.tlfq.ulaval.ca $>$.

170 
être regroupés selon des critères politiques. Ceux-ci sont retenus lors des Sommets de la Francophonie où on tient compte de différents représentants des communautés ou minorités francophones dans des pays bilingues comme le Canada (la délégation canadienne en tant que membre et les gouvernements du Québec et du Nouveau-Brunswick) ou la Belgique (Le Royaume de Belgique et la Communauté française de Belgique).

Le terme francophonie a été utilisé avec beaucoup de réticence jusque dans les années 80 car il comportait une connotation néocolonialiste. Ce n'est qu'en 1986 qu'il est utilisé sans complexe par F. Mitterrand lors du ler Sommet de la Francophonie. Il est assez couramment remplacé par le terme Espace francophone qui exprime plutôt une notion de contact et d'échange entre les pays.

Pour terminer cette brève évocation des principaux aspects de la francophonie rappelons que le terme Francophonie écrit avec un $\mathrm{F}$ majuscule désigne l'ensemble d'organismes, de Gouvernements et des pays qui utilisent le français dans leurs échanges, que ce soit lors des Sommets ou des contacts individuels. Depuis 1986 les Etats membres de la Francophonie se réunissent tous les deux ans dans des sommets dans lesquels sont discutés des politiques communes af in de favoriser le développement des pays et les échanges d'expériences dans les domaines politique, économique, culturel entre autres. Ces sommets sont importants pour la Francophonie car ils ont permis d'établir un réseau d'échange dynamique entre les états membres ce qui attire de plus en plus d'autres gouvernements ou pays qui n'ont pas le français comme langue of ficielle mais qui ont des liens historiques ou culturels avec la France ou les autres pays. Ainsi, par exemple, la Francophonie accueille en son sein le Cambodge, la Roumanie et la Bulgarie en 1991, la Moldavie et Saint-Thomas-et-Prince en 1995, l'Albanie et la Macédoine en 1999. Ces sommets marquent aussi l'évolution du réseau qui passe d'un ethnocentrisme français au principe de la diversité culturelle, ce qui a été confirmé au VIII Sommet de Moncton. 


\section{La langue française et ses conflits avec la francophonie}

La langue française a un rôle paradoxal dans la francophonie car elle est l'élément unificateur qui lui a donné naissance mais elle constitue également un élément conflictuel qui peut mener jusqu'à la rupture. La plupart des pays francophones entretiennent, en fait, une relation très passionnelle avec le français qui oscille entre l'amour et la haine.

Tout au long de son histoire la langue française s'est imposée par la force non seulement en France, provocant l'éradication des langues ou des dialectes régionaux mais aussi en Belgique où elle a prédominé sur le flamand (néerlandais) jusqu'au milieu du XIXème siècle. Ce phénomène était dû surtout au fait que les classes nobles refusaient d'utiliser le "patois" populaire en Flandre. Grâce à une économie florissante le flamand renverse la situation et s'impose comme la seule langue en Flandre reléguant le français à la deuxième place $(41 \%$ de francophones) dans le pays. Dans les autres régions du monde l'expansion a été d'abord d'ordre démographique et par la suite d'ordre économique et militaire lors des conquêtes coloniales. Ceci s'est traduit dans la plupart des cas par l'implantation, dans les territoires conquis, de l'enseignement en français et d'instances administratives au détriment des langues nationales. Ce processus a été relativement favorisé par le fait que la plupart des langues, spécialementen Afrique, étaient utilisées uniquement dans un registre oral et non pas textuel.

La France a imposé au monde entier non seulement sa langue mais aussi son prestige au point de déclencher une polémique même au sein des pays qui ont le français comme langue maternelle. C'est le cas de la Belgique et du Canada. Cette controverse s'exprime particulièrement dans la littérature. En effet, les écrivains belges s'affrontent selon deux thèses, à savoir si les écrits littéraires belges doivent être désignés comme "Littérature belge d'expression française ou comme lettres françaises de Belgique". Pour les milieux cultivés de 
Belgique la littérature belge n' existe pas. Cette thèseest particulièrement défendue par certains écrivains qui se sont regroupés sous le nom de Groupe du Lundi. Ils affirment que la littérature de Belgique fait partie intégrante de la France. Cette vision est due surtout à la diversité de tempéraments imposée par le métissage subi par le peuple au cours de son histoire. En effet, la Belgique a connu les invasions germaniques, les occupations espagnole et autrichienne, entre autres. Actuellement elle est partagée entre trois communautés d'origines différentes : les Wallons d'origine celte, les Flamands d'origine germanique et une petite minorité d'allemands.

D' autres écrivains considèrent que ce mélange de cultures donne aux écrivains belges une vision propre de l'univers et donc une manière particulière de penser et d'écrire ce qui les différencie des écrivains français. Aussi depuis 1920 la conception d'une littérature nationale s'impose peu à peu, véhiculée par certaines revues littéraires.

En 1938, Gustave Charlier dans un étude historique, constate que :

Nous tenons, pour notre compte, que nos lettres françaises, sans constituer une littérature nationale, au sens strict du mot, possèdent néanmoins une unité réelle, qui ne permet pas de les confondre tout à fait avec la littérature de la France de Louis XIV, de Napoléon III ou de M. Albert Lebrun. ${ }^{2}$

En effet beaucoup d'oeuvres belges s'inspirent du passé national et des légendes du terroir. Les écrivains montrent souvent un certain goût pour le fantastique qu' ils distillent en filigrane dans leurs oeuvres. Contrairement aux autres cultures francophones pour qui la langue française est l'instrument qui leur permet de revendiquer leur identité, certains Belges semblent plutôt vouloir renoncer à leur spécificité. Ceci paraît une aberration d'autant plus que le seul lien qui les unit à

2. $\quad$ Gustave Charlier, Les lettres française de Belgique (Bruxelles: La Renaissance du livre, 19.38) 7. 
la France c'est leur langue commune. Pourquoi donc veulent-ils être assimilés à la France littéraire ? Cette attitude est compréhensible par le fait que les écrivains belges désirant se faire connaître doivent passer par Paris qui continue d'écraser de son prestige toutes les activités artistiques.

En ce qui concerne le Canada, la controverse se présente autour de deux axes : celui de la langue et celui de la littérature. A cet égard "Notre langue", texte de Rex Démarchais, est une parfaite illustration car il expose les arguments des deux tendances qui s'affrontent. ${ }^{3} \mathrm{~A}$ l'instar de Démarchais certains écrivains, principalement au début de la littérature canadienne, penchent plutôt vers l'utilisation d'un français correct, scolaire car c'est lui qui leur permet de défendre leur identité et leur origine. Ainsi que l'explique Louis Dantin :

Une littérature ne brise pas impunément avec tout son passé...Notre race est née, a grandi, liée au langage de la France, défendue et sauvée par lui. Le français qu'elle parlait, c'est ce qui, plus que tout le reste, lui a gardé son individualité, la permanence distincte parmi les autres races qui l'entouraient et la débordaient. ${ }^{4}$

Ceci s'explique, rappelons-le, par le fait que les Canadiens sont des français qui ont émigré au Canada et qu'ils restent attachés à la France qu'ils considèrent comme "la mère patrie" au point de perpétuer, dans leur coeur, un culte certain. La langue française est considérée comme un patrimoine. Le fait de ne pas la parler ou de ne pas l'écrire correctement serait une sorte de trahison envers la terre de leurs ancêtres et par la même occasion cela provoquerait l'anéantissement de leur identité si âprement défendue. En effet, le Québec a pris certaines mesures en faveur de la langue française comme par exemple

3. Rex Démarchais, "Notre langue", Amérique française (1948).

4. Louis Dantin, Glosses critiques (Montréal: Ed. Albert Lévesque, 1931) 170-175. 
La Charte de la langue française en 1977, la Loi 101 (limitant l'usage de l'anglais dans des activités publiques et commerciales) af in d'éviter qu'elle se dilue avec les apports de l'anglais ou d'autres langues.

D'autres écrivains prônent plutôt l'utilisation d'une langue du terroir : le "joal", une sorte de créole plus adapté à leur réalité et à leur histoire. Mais ils craignent de rendre incompréhensible la communication autant vis-à-vis des Canadiens que des autres pays francophones. La littérature connaît la même situation que la littérature belge. Les écrivains sont divisés en ce qui concerne la définition de leurs écrits entre l' appellation de "Littérature canadienne d'expression française" ou bien "Littérature Québécoise" car de nombreux auteurs considèrent que le Canada n'a pas d'identité bien définie avec laquelle ils pourraient s'identifier. Rappelons, en effet, que les francophones au Canada constituent une minorité (6 millions contre 250 millions d'anglophones). Les Québécois ont une spécificité de par leur origine française. Tout au long de leur histoire ils ont dû se battre pour ne pas se laisser engloutir par la culture anglophone et pouvoir conserver leur identité et leur spécificité. C'est ce qu'affirme Ivanhoé Beaulieu :

ce qui s'écrit dans mon pays ne s'assimile en rien à cette entité abstraite qui est le Canada. Québécois suppose un territoire, Québécois suppose aussi la reconnaissance d'une histoire, d'une conscience, et même d'une esthétique propre. ${ }^{5}$

A cet effet rappelons que la province du Québec est la seule à avoir la langue française comme langue officielle, les autres étant bilingues ou anglophones. La question se pose autrement pour les autres pays francophones car la langue française représente avant tout la langue du colonisateur qui a étéimposée aux différentes populations. Elle permettait à la France de garder l'unité de son empire. Et pourtant, l'ironie du sort a fait qu'elle est devenue, après l'indépendance, le

5. Marcel Dugas, Littérature canadienne (Paris: Firmin-Didot et Cia. 1929) 1-3. 
moyen de revendication identitaire par excellence, ce qui a posé aux écrivains un cas de conscience: dans quelle langue écrire ? Ils devaient contribuer à la construction de leurs nouveaux Etats, mais pour ce faire ils devaient utiliser le français tout d'abord parce qu' ils écrivaient pour "L'Autre" afin d'assouvir le besoin de reconnaissance de leur culture, puis parce que dans le contexte colonial les seuls lecteurs possibles étaient une élite qui parlait français.

Ainsi donc les écrivains ont reproduit les modèles français. Cette attitude a été jugée servile, à tort, car comme l'explique Edouard Glissant ${ }^{6}$ la culture du maître avait été assimilée et même intériorisée. Cet avis est partagé par Aimé Césaire qui, lors d'une rencontre avec des élèves latinoaméricains de l'ISEF en mars 2002 a expliqué qu'il écrivait en français parce que c'était la seule langue qu'il savait écrire. Ces écrivains se trouvaient dans une situation conflictuelle puisqu'il leur fallait d'abord retrouver leurs racines pour pouvoir les faire connaître au colonisateur. Pour bien la transmettre ils devaient écrire dans une langue comprise par "l'Autre". Rappelons que les seules images que possédaient les colonisateurs de ces terres lointaines avaient été dépeintes par des écrivains ou des colons français qui avaient créé une sorte de littérature exotique selon leur vision occidentale.

$\mathrm{Au}$ fur et à mesure où les identités se définissent, naissent différents mouvements comme celui de "La Négritude" ébauché par Aimé Césaire, Léopold Sedar Senghor et Léon-Gontran Damas au début des années 30. Leur but était d'affirmer l'existence d'une civilisation noire dont la grandeur avait été brimée, niée par le monde occidental et par la même occasion leur fierté par rapport aux valeurs culturelles noires. Ils dénonçaient et critiquaient durement la colonisation française. Comme ces écrivains avaient reçu une éducation française, ils avaient conçu cette notion de Négritude avec une vision trop occidentalisée puisqu'elle effaçait les différences entre les divers

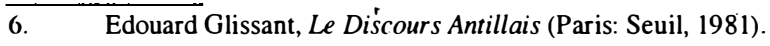


pays noirs sans tenir compte ni de leur contexte géographique ni de leur situation politique. Et pourtant elles existent car qu'est-ce qui peut réunir dans un même ensemble littéraire un Patrick Chamoiseau, un Ferdinad Oyono ou un Cheikh Hamidou Kane ? et plus encore s'ils sont comparés avec des écrivains de l'Afrique du Nord ou du Liban tels que Tahar Ben Jelloun, Albert Memmi ou Andrée Chedid. Rappelons à ce propos que, contrairement aux anciennes colonies et protectorats français, les Antilles (La Martinique, la Guadeloupe et la Guyane française) subissent encore le poids de la France car elles en forment partie intégrante. Tout comme le Canada, ces cultures sont tributaires des conditions de vie particulières, ce qui va conditionner leur spécificité. C'est le constat fait par Edouard Glissant vers les années 60 , qui considère que les Antilles se débattent dans une crise identitaire. Il va opposer au concept de "La Négritude" celui de "L'Antillanité" qui tiendra compte des contraintes géographiques des Antilles et des dégâts sociaux causés par la colonisation. Il faut, dit-il, "se réapproprier l'espace antillais"7 pour pouvoir reconstituer l'identité antillaise, rappeler aux Antillais l'histoire de l'esclavage et des plantations. Al'instar d'Edouard Glissant, Raphaël Confiant confirme, lors d'une rencontre avec des élèves latinoaméricains du DEA de l'ISEF en mars 2002, que les Antillais ont des difficultés à se définir pour deux raisons. D'abord par la diversité des cultures qui y cohabitent : des colons blancs, des noirs, des indiens et des mulâtres. Les colons blancs - "les Békés" - ont imposé facilement leur culture lorsqu'ils se sont implantés dans les îles non seulement parce qu'ils étaient les plus forts économiquement mais aussi parce les noirs qui ont été emmenés comme esclaves étaient pour la plupart des enfants de 13-14 ans qui venaient d'ethnies différentes et qui ne parlaient pas les mêmes langues. Ils ont adopté le créole pour pouvoir communiquer entre eux. Les indiens venus après l'abolition de l'esclavage sont méprisés donc sont peu ou pas du tout intégrés dans la société. Les mulâtres se sont

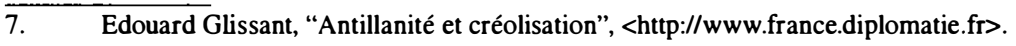


plutôt rapprochés des blancs et ont donc adopté leur manière de vivre. Ils seront les premiers à apprendre à lire et à écrire. Outre cette mosaïque culturelle, les Antilles ont été marquées par une colonisation réussie qui a imposé dans l' esprit des antillais la négation de tout ce qui n'est pas blanc. Depuis l'époque de l'esclavage jusqu'à 1952 tout ce qui était noir était méprisé. Aussi les antillais essayaient-ils de devenir blancs par tous les moyens, même en imitant les Békés. Aussi lorsqu'Aimé Césaire publie son manifeste de la Négritude celui-ci n'a pas le même succès qu'il a en France. Ceci explique la servilité dont on les accuse. Il est évident que dans ce contexte il est difficile pour les écrivains de se démarquer de la France comme entité à part entière. Patrick Chamoiseau se questionne à ce sujet dans le premier paragraphe de son essai Ecrire en pays dominé : Comment écrire alors que ton imaginaire s'abreuve, du matin jusqu'aux rêves, à des images, des pensées, des valeurs qui ne sont pas les tiennes ? $^{8}$

La quête identitaire des Antillais se poursuit dans les années 80 avec le mouvement littéraire de "la Créolité" qui a une démarche beaucoup plus intellectuelle. Par le biais de l'écriture Jean Bernabé, Patrick Chamoiseau et Raphaël Confiant essaient de reconstruire l'histoire antillaise avec la diversité des peuples qui la composent. Ils manifestent cependant la même difficulté que les Canadiens et les Belges à concevoir l'existence d'une littérature antillaise et ils le confirment dans Eloge de la créolité. ${ }^{9}$

Le rapport des Africains avec la langue française est plutôt à la rupture. Comme pour les Antillais, le français est avant tout la langue du colonisateur, celle qui a brimé pendant longtemps leurs langues maternelles et leurs cultures et elle continue de dominer leur vie dans le domaine de l'enseignement et de la communication. Deux raisons expliquent cet situation. D'abord parce que dans chaque pays coexiste

\footnotetext{
8. Patrick Chamoiseau, Ecrire en pays dominé (Paris: Gallimard, 1997) 1.

(). Jean Bernabé, Patrick Chamoiseau, Raphaël Confiant, Eloge de la créolité (Paris: Gallimard, 1989).
} 
une grande diversité d'ethnies et de langues, donc le français leur permet de communiquer entre elles. Cette mosaïque de peuples remet en question jusqu'à un certain point le terme de la Négritude car il n'y a pas une mais plusieurs cultures noires avec leurs spécificités. En outre comme il a été mentionné auparavant ces langues ne sont pratiquées qu'à l'oral, elles ne sont pas écrites. C'est, d'ailleurs à cause de cela que certains pays ont instauré une politique de scolarisation de masse qui n'a pas été bien réussie en raison du manque de moyens. Rappelons aussi que le français n'est parlé, dans la plupart des pays, que par une minorité ( 1 \% en Côte d'Ivoire, 6 \% au Congo, $4 \%$ au Burundi, $13 \%$ au Bénin, etc. ${ }^{10}$ ). La deuxième raison réside dans le fait que, même si on est opposé à la langue de l'envahisseur, il faut se l'approprier pour acquérir la connaissance et la maîtrise technique qui marquent sa supériorité. Cette appropriation leur permettra non seulement de mieux les connaître mais aussi de se libérer de leur domination. C'est en tout cas ce qu' expriment certains écrivains à travers leurs personnages comme par exemple la Grande Royale dans le roman L'aventure ambigüe de Hamidou Kane qui confirme cette conviction

Notre grand-père, ainsi que son élite, ont été défaits. Pourquoi ? Comment ? Les nouveaux venus seuls le savent. Il faut le leur demander; il faut aller apprendre chez eux l'art de vaincre sans avoir raison. ${ }^{11}$

Malheureusement au lieu de s'affranchir, ils ont provoqué leur perte car l'école a bouleversé les croyances et la vision de la société africaine pour imposer les siennes. D' ailleurs, ainsi que le proclame Cheikh Hamidou Kane dans le même livre :

10. TLFQ-Université Laval Québec (2002), "La francophonie dans le monde". Consulté en avril 2002 : <http://www.tlfq.ulaval.ca/axl/francophonie/francophonieacc.htm〉.

11. Cheikh Hamidou Kane, "L'aventure ambigüe" (Paris: Juillard : Collection 10/18 - 1961) 47. 
L'école étrangère est la forme nouvelle de la guerre que nous font ceux qui sont venus...L'école où j'envoie nos enfants tuera en eux ce qu'aujourd'hui nous aimons et conservons avec soin, à juste titre. Peut-être notre souvenir lui-même mourra-t-il en eux. Quand ils reviendront de l'école, il en est certains qui ne nous reconnaîtront pas. ${ }^{12}$

C'est le constat fait par les écrivains déçus de leurs dirigeants qui, par leur attitude, ont provoqué la faillite de leur société. Ils critiquent durement ces nouveaux maîtres avides de pouvoir et de richesse non pas pour la tribu mais pour eux-mêmes à l'instar des conceptions individualistes des sociétés occidentales. Rappelons qu'un certain nombre de dirigeants africains ont fait des études en France. Tot comme Samba Diallo, personnage principal de L'aventure ambigüe, déjà cité auparavant, ils ont perdu leurs repères africains et leurs valeurs tribales.

Contrairement aux sociétés occidentales qui sont individualistes l'Afrique conçoit l'existence par rapport au groupe. Ce phénomène est évident dans beaucoup d'oeuvres africaines ou maghrebines ou le "je" est absent. Néanmoins le français pur de France ne convient pas tout à fait aux pays africains, non seulement par le contexte socioculturel mais aussi linguistique. La langue française ne peut donc pas toujours traduire la réalité sociale des pays africains qui ont plutôt une culture tribale régie soit par des patriarcats soit par des matriarcats, ce qui va déterminer entre autres le dégré de parenté. Ainsi que l'explique Dominique Rolland dans son article Frère ${ }^{13}$ si le degré de parenté est déterminé par le père on appellera "frère" autant les frères que les cousins issus de la lignée paternelle. Par contre les cousins ou les autres parents issus de la lignée maternelle seront désignés par d' autres termes. En Afrique les termes cousin, oncle, tante n'ont aucun

12. Hamidou Kane, 47-57.

13. Dominique Rolland, "Frère", Le Français dans le Monde, 302, 25. 
sens par rapport à leur conception sociale. Nombreux sont les exemples qui démontrent cette différence de conception et qui peuvent provoquer des équivoques entre les Africains et les occidentaux. Ainsi que l'explique Ahmadou Kourouma ${ }^{14}$, dans l'entretient qu'il a accordé à la revue Diagonales, pour pouvoir faire vivre son personnage et le rendre vraisemblable par rapport au peuple malinké il lui fallait adapter le français à la structure et au rythme de la culture malinké. Ce changement dans la conception du roman est dû au fait qu'il n'écrit plus seulement pour "l'Autre" mais aussi pour les Africains. Ceci lui permet d'évoquer leurs traditions, leurs racines, de les remettre dans le contexte africain et même de leur réapprendre leur africanité car ils s'y reconnaissent.

Enfin, pour terminer ce bref tour des conflits vécus dans l'espace francophone, évoquons le cas des pays maghrebins. Depuis leur indépendance entre 1952 et 1962 le statut du français a commencé à changer. Quel que soit le pays, le français représente pour beaucoup de maghrebins la langue du colonisateur. Mais, comme les arabes désiraient renouer avec leurs traditions, ils ont instauré une politique 'd'arabisation qui a relégué le français, aux alentours des années 80 , au rôle de langue à statut privilégié ou de langue seconde (car elle est encore très présente dans l'enseignement). Deux tendances s' affrontent: ceux qui sont en faveur de l'arabisation et ceux qui continuent de penser que le français est la langue de la science et du savoir, qu'elle est utile et enrichissante et de ce fait mène vers la modernité. Ils ne veulent pas être isolés d'un monde qui évolue sans cesse. En ce qui concerne la littérature, certains écrivains - exaspérés par l'ethnocentrisme français et l'écrasante domination exercée sur les différents pays de la francophonie - ont décidé de rompre non pas avec la langue mais avec la francophonie. Tel est le cas de l'auteur algérien Rachid Boudjedra ${ }^{15}$ qui l'explique à Claude Revil dans une interview

14. Ahmadou Kourouma, Diagonales $N^{\circ} 7$ (juillet 1988).

15. Claude Revil, Diagonales $\mathrm{N}^{\circ} 2$ (juillet 1987). 
publiée dans la revue Diagonales. Son attitude est compréhensible mais il se coupe d'une partie des lecteurs non seulement occidentaux mais du monde francophone qui ne parlent pas arabe et c'est dommage. Au départ, lorsqu'ils ont commencé à écrire en français, leur démarche était de faire renaître les traditions arabo-musulmanes et de les faire reconnaître par les occidentaux. Néanmoins pour certains écrivains comme le Marocain Tahar Ben Jelloun, le fait d'écrire en français lui permet de dire ce qu' il ne pourrait pas dire en arabe parce que ce serait mal perçu par les siens. Dans ses oeuvres, il critique fortement sa propre culture. Quoi qu'il en soit, dans ces pays le français est surtout une langue de communication entre les communautés arabes et berbères qui y cohabitent, malheureusement sous une tension certaine.

\section{L'interculturel}

La francophonie devient un espace interculturel au moment où les peuples qui la composent commencent à prendre conscience de leur spécificité. Elle va donner naissance à des nouvelles écritures d'une grande diversité et cela bien qu'elles aient en commun l'utilisation de la langue française, leur histoire coloniale, l'oralité des récits et des thèmes comme l'esclavage, la recherche des origines, les traditions, etc: Au fur et à mesure où les identités se définissent, naissent différents mouvements dont le but est d'affirmer l'existence de diverses civilisations qui partagentl'espace francophone. Desécrivains tels que Raphaël Confiant essaient de décrire dans leurs oeuvres les réalités de leurs îles qui, à déf aut d'une culture ancienne, les démarquent plus que tout autre élément de la France.

Cette prise de conscience s'affirme peu à peu avec les nouvelles générations puisque les écrivains s'expriment en créole et ne s'adressent plus à "l' Autre" mais à leur peuple. Il ne s'agit plus de faire accepter leurs différences mais de faire découvrir à leurs compatriotes leur Antillanité. D'ailleurs, lorsque Raphaël Confiant a écrit son premier roman en français il a éprouvé, selon ses propres 
mots, le sentiment d'avoir trahi sa propre culture. Et pourtant ses oeuvres ont été d'une grande importance pour la littérature antillaise d'expression française car elles ont su imposer sa différence pas seulement dans le contenu mais aussi dans le style empreint d'une richesse de mots et d'expressions souvent crées par lui et d'un humour et d'une ironie cinglants. Ses écrits sont paradoxaux car d'un côté il semble manifester l'insécurité linguistique propre à beaucoup de citoyens de la francophonie (il insère souvent dans ses écrits des explications par rapport aux termes utilisés tout en confirmant sa connaissance du sens des mots), d'un autre côté, à bien étudier ses textes, il manifeste une sorte de mépris et même de défi par rapport au français dans le sens où il affirme sa maîtrise du français mais en revendiquant son droit de l'adapter à sa sensibilité et à sa réalité antillaise. C'est un appel à l'interculturel qu'il lance. Les écrivains antillais établissent ainsi un dialogue des cultures dans leur propre espace et dans ce sens ils se rapprochent de la tendance moderne de la francophonie.

Le même processus d'ouverture vers l'autre est entamé par Ahmadou Kourouma et sera suivi par d'autres écrivains africains. Ainsi les différents pays commencent à enrichir le français avec de nouveaux mots, des proverbes, des expressions d'une grande vitalité, ce qui a conduit à l'édition de dictionnaires tels que Le Dictionnaire Universel Francophone, Le Dictionnaire Universel Afrique, etc. C'est un fait relevant car grâce à cela ces écrivains ont perdu leur insécurité par rapport au français de France et en même temps, ils ont une fois pour toutes marqué leurs spécificités et leurs identités.

\section{Conclusion}

Le français est une langue qui déchaîne les passions et qui a cultivé tout au long de son histoire les conflits. Il a surtout permis à la culture française de rayonner dans le monde entier en assurant ainsi sa pérennité. Même si, dans les dernières années, il a vécu un certain 
déclin dans les pays francophones au profit soit de l'anglais soit des langues locales comme le créole ou certaines langues africaines, il gagne du terrain dans des pays non francophones. Des milliers de personnes l'étudient non seulement dans des institutions privées comme l'Alliance Française mais aussi dans desétablissements publics, constituant ainsi un vaste réseau francophile. C'est un fait relevant qui ouvre la voie à la France pour étendre son influence autant dans le domaine culturel que dans les domaines commercial, technique et scientifique. Mais la France n' est plus le seul vecteur de l'enseignement du français. Les Canadiens jouent de plus en plus un rôle dynamique dans ce domaine. La francophonie ne s'exerce plus à sens unique: de la France vers les autres pays francophones car depuis des années les identités des différents pays s'affirment et se font reconnaître surtout grâce à la littérature. Dans ce contexte le terme francophonie ne convient plus à cet ensemble pour deux raisons. D'abord parce qu'il rappelle le colonialisme, puis parce que il n'y a pas une seule francophonie mais plusieurs dans le sens de la multiplicité des cultures. Ces pays ont certainement des points communs, tels que l'utilisation de la langue française et leur passé colonial mais ils appartiennent à différentes zones géographiques, possèdent des traditions culturelles et des expériences historiques différentes et donc des sensibilités propres.

Comme ce concept de Culture est devenu plus important que la langue, même des pays comme le Vietnam qui avaient coupé leurs relations historiques avec la France reprennent contact dans une relation d'égal à égal. Huu Ngoc, auteur vietnamien de plusieurs ouvrages de littérature le confirme à Bernard Magnier. ${ }^{16}$ On a souvent cru que la littérature de langue française ne survivrait pas à la période coloniale. Trente ans après on constate, au contraire, qu' une multitude de romans, de poèmes, de nouvelles, de pièces de théâtre...écrits en français voit le jour dans les pays du Maghreb, en Afrique subsaharienne,

16. Bernard Magnier, Diagonales $N^{\circ} 43$ (août 1997). 
aux Caraïbes et dans l'Océan Indien pour insuffler une nouvelle dynamique de l'interculturel.

Ces diverses littératures francophones se caractérisent tout d'abord par un bilinguisme qui joue un rôle essentiel. La plupart des écrits antillais, africains et maghrébins sont parsemés de tournures syntaxiques, d'images, de métaphores propres à leurs langues maternelles. Elles sont marquées en outre par l'oralité et privilégient le fantastique, l'onirique, l'irréel, l'humour et les rêveries dans le but de dénoncer des réalités insupportables, inadmissibles. C' est la richesse de l'oeuvre de l'écrivain Tahar Ben Jelloun qui dénonce dans ses romans L'enfant de sable et La nuit sacré, entre autres, le sort cruel réservé à la femme arabe dans les pays maghrébins. Ainsi donc, malgré les années de colonialisme et la domination exercée par la France et le français certains écrivains ont su reconquérir leurs racines pour enrichir tout le monde francophone. 